\title{
A Convenient Route for Synthesis and Antimicrobial Evaluation of Bis (diimino Benzothiazolo Pyrimido Pyrimidines)
}

\author{
BALASAHEB D. KALYANKAR, PRASHANT N. UBALE and SAMBHAJI P. VARTALE* \\ Department of Chemistry, Yeshwant Mahavidyalaya Nanded - 431 602, India. \\ ${ }^{*}$ Corresponding author E-mail: spvartale @ gmail.com \\ http://dx.doi.org/10.13005/ojc/300448
}

(Received: August 07, 2014; Accepted: September 26, 2014)

\begin{abstract}
Guanidine hydrochloride (1) on treatment with bis(methylthio)methylene malononitrile (2) in N,N-dimethyl formamide (DMF) and catalytic amount of anhydrous potassium carbonate gives diimino pyrimido pyrimidine (3). The later were further reacted with various substituted 2-amino benzothiazoles (4) to gives bis (diimino benzothiazolo pyrimido pyrimidines) (5a-g). All these synthesized compounds were screened for their antimicrobial activity.
\end{abstract}

Key words: Bis(methylthio)methylene malononitrile; Guanidine hydrochloride; $\mathrm{N}, \mathrm{N}$-dimethyl formamide (DMF) and Potassium carbonate.

\section{INTRODUCTION}

Pyrimido pyrimidine and its derivatives are interesting class of nitrogen containing heterocyclic compounds which are widely found in bio-organic and medicinal chemistry with application in drug discovery. Pyrimido $[4,5-d]$ pyrimidines, pyrido $[2,3-$ d] pyrimidines are important class of annulated uracils which have biological importance ${ }^{1}$ because of their connection with purine pyrimidine system ${ }^{2}$ . Some of them exhibit significant biological and pharmacological activities such as antifolate activity $^{3}$, antibacterial activity ${ }^{4-7}$, tyrosine kinase activity ${ }^{8}$. Numerous reports have appeared in the literature for preparation of fused heterocycles. Derivatives of pyrimido pyrimidine are useful as bronchodilators $^{9}$, vasodilators ${ }^{10}$, antiallergic ${ }^{11}$ and hypersensitive agent ${ }^{12}$, more over they display potent inhibitory properties regarding tyrosine kinase domain of epidermal growth factor receptor $^{13}$. Various researcher reported pyrimido pyrimidine explore the antitumor ${ }^{14}$, antiviral ${ }^{15}$, antioxidant ${ }^{16}$, antifungal ${ }^{17}$ and hepatoprotective activities $^{18}$. 
A survey of literature reveals that few references are available on the synthesis and biological activity of heterocycles containing a benzothiazole fused with pyrimidine ring ${ }^{19-22}$. Patrick Jimonet and his research group ${ }^{23}$ reported the synthesis and pharmacological activity of 3substituted -2-imino benzothiazolines. These compounds were found to be three times more potent than 6-(trifluoromethoxy)-2benzothiazolamine (Riluzole), a blocker of excitatory amino acid mediated neurotransmission. These observations stimulated us to considerable interest for the synthesis of new compounds in which the imino pyrimidine ring is fused through a nitrogen atom with another biological active nucleus such as benzothiazole. Recently our research group reported synthesis of pyrimido thiazine and its derivatives ${ }^{24-26}$.

The present review is devoted exclusively to the synthesis of diimino pyrimido pyrimidine and its reaction with 2-Amino benzothiazoles. In present manuscript we emphasize the synthetic routes to heterocyclic compounds via diimino pyrimido pyrimidine with evaluation of their anti microbial activity.

\section{MATERIALAND METHODS}

All the chemicals used in the present study are of analytical grade purchased from Himedia chemical co. All reactions were monitored by thin layer chromatography, were carried out on $0.2 \mathrm{~mm}$ silica gel-C plates, the spots were examined under UV light chamber. Melting points of synthesized compounds were determined by Electrothermal IA 9000 SERIES digital melting point apparatus and were uncorrected. Solvents were purified according to standard procedure. Infrared spectra were recorded in Nujol or as potassium bromide pallets on infrared spectrophotometer, nuclear magnetic resonance spectra were obtained on Brukner advance spectrophotometer $400 \mathrm{MHz}$, Mass spectra were recorded on FT-VC-7070 H Mass spectrometer using the El technique at $70 \mathrm{eV}$. All the reactions were carried out under ambient atmosphere. Elemental analysis was performed on a Heraeus $\mathrm{CHN}-\mathrm{O}$ rapid analyser.

\section{General procedure}

2,6-dihydro-2,6-diimino-4,8-bis(methylthio)-1Hpyrimido[1,2-a]pyrimidine-3,7-dicarbonitrile (3)

A mixture of guanidine hydrochloride (1) $(0.01 \mathrm{~mol})$ and bis(methylthio)methylene malononitrile (2) $(0.02 \mathrm{~mol})$ in $20 \mathrm{ml}$ of $\mathrm{N}, \mathrm{N}$-dimethyl formamide (DMF) and anhydrous potassium carbonate $(10 \mathrm{mg})$ was refluxed for 12 hours. After completion of reaction, the reaction mixture was cooled to room temperature and poured in to ice cold water. The seperated solid product was filtered , washed with water and recrystalized from N,Ndimethyl formamide-ethanol mixture to give pure (3).

Yellow powder, Yield 62\%, M.P. 190-192 ${ }^{\circ} \mathrm{C}$. IR $\left(\mathrm{KBr} / \mathrm{cm}^{-1}\right) 3416 \mathrm{~cm}^{-1}(=\mathrm{NH}), 3380 \mathrm{~cm}^{-1}(-\mathrm{NH})$, $2205 \mathrm{~cm}^{-1}(\mathrm{CN}), 1680 \mathrm{~cm}^{-1}(\mathrm{C}=\mathrm{N}-)$ : ${ }^{1} \mathrm{HNMR}(400$ $\left.\mathrm{MHz}, \mathrm{DMSO}-\mathrm{d}_{6}\right): \delta=2.1(\mathrm{~s}, 1 \mathrm{H},-\mathrm{NH}), 2.7(\mathrm{~s}, 6 \mathrm{H}$, $\left.\mathrm{SCH}_{3}\right), 8.1(\mathrm{~s}, 2 \mathrm{H},=\mathrm{NH}): \mathrm{El}-\mathrm{MS}(\mathrm{m} / \mathrm{z}: \mathrm{RA} \%): 303\left(\mathrm{M}^{+}\right)$. Anal. Calcd for $\mathrm{C}_{11} \mathrm{H}_{9} \mathrm{~N}_{7} \mathrm{~S}_{2}: \mathrm{C}: 43.56, \mathrm{H}: 2.97, \mathrm{~N}: 32.34$, found: C:43.31, H:2.84, N:32.21.

\section{Diimino bis benzothiazolo pyrimido pyrimidines (5a-g)}

A mixture of (3) $(0.001 \mathrm{~mol})$ and independently varios substituted 2-amino benzothiazoles (4) like 2-amino benzothiazole, 2amino-6-methyl benzothiazole, 2-amino-6-methoxy benzothiazole, 2-amino-6-bromo benzothiazole, 2amino-4,6-dimethyl benzothiazole, 2-amino-5,6dichloro benzothiazole, 2-amino-5-nitro benzothiazole $(0.002 \mathrm{~mol})$ in $10 \mathrm{ml}$ of $\mathrm{N}, \mathrm{N}$-dimethyl formamide (DMF) and anhydrous potassium carbonate $(6 \mathrm{mg})$ was refluxed for 8 hours. The reaction mixture was cooled to room temperature and poured in to ice cold water. The separated solid product was filtered, washed with water and recrystalized from $\mathrm{N}, \mathrm{N}$-dimethyl formamide-ethanol mixture to give pure (5a-g).

\section{$5 a$}

Brown powder, Yield $68 \%$, M.P. $215^{\circ} \mathrm{C}$. IR $\left(\mathrm{KBr} / \mathrm{cm}^{-1}\right) 3450 \mathrm{~cm}^{-1}(=\mathrm{NH}), 3365 \mathrm{~cm}^{-1}(\mathrm{NH}), 1690$ $\mathrm{cm}^{-1}(\mathrm{C}=\mathrm{N}) .{ }^{1} \mathrm{HNMR}\left(400 \mathrm{MHz}, \mathrm{DMSO}-d_{6}\right): 6.2-6.7$ (m, 8H, Ar-H), 8.5 (s, 4H, =NH): El-MS (m/z: RA\%): $507\left(\mathrm{M}^{+}\right)$. Anal. Calcd for $\mathrm{C}_{23} \mathrm{H}_{13} \mathrm{~N}_{11} \mathrm{~S}_{2} \mathrm{C}: 54.43, \mathrm{H}$ : 2.56, N: 30.37, found; C: $54.27, \mathrm{H}: 2.51, \mathrm{~N}: 30.15$. 
$5 b$

Brown powder, Yield $71 \%$, M.P. $205^{\circ} \mathrm{C}$. IR $\left(\mathrm{KBr} / \mathrm{cm}^{-1}\right) 3432 \mathrm{~cm}^{-1}(=\mathrm{NH}), 3372 \mathrm{~cm}^{-1}(\mathrm{NH}), 1660$ $\mathrm{cm}^{-1}(-\mathrm{C}=\mathrm{N}-)$. ${ }^{1} \mathrm{HNMR}\left(400 \mathrm{MHz}, \mathrm{DMSO}-d_{6}\right):^{\prime}=2.2($ $\left.\mathrm{s}, 6 \mathrm{H}, \mathrm{Ar}-\mathrm{CH}_{3}\right), 6.1-6.8(\mathrm{~m}, 6 \mathrm{H}, \mathrm{Ar}-\mathrm{H}), 9.1$ (s, 2H, $=\mathrm{NH})$ : El-MS (m/z: RA\% ): $535\left(\mathrm{M}^{+}\right)$. Anal. Calcd for $\mathrm{C}_{25} \mathrm{H}_{17} \mathrm{~N}_{11} \mathrm{~S}_{2} \mathrm{C}: 56.07, \mathrm{H}: 3.17, \mathrm{~N}: 28.73$; found: $\mathrm{C}: 56.01, \mathrm{H}: 3.08, \mathrm{~N}: 28.45$.

$5 c$

Brown powder, Yield $66 \%$, M.P. $224^{\circ} \mathrm{C}$. IR $\left(\mathrm{KBr} / \mathrm{cm}^{-1}\right) 3440 \mathrm{~cm}^{-1}(=\mathrm{NH}), 3342 \mathrm{~cm}^{-1}(\mathrm{NH})$, $1667 \mathrm{~cm}^{-1}(-\mathrm{C}=\mathrm{NH}-):{ }^{1} \mathrm{HNMR}\left(400 \mathrm{MHz}, \mathrm{DMSO}-d_{6}\right): \delta$ = 3.6-3.8 ( s, $\left.6 \mathrm{H},-\mathrm{OCH}_{3}\right), 6.8-7.5(\mathrm{~m}, 6 \mathrm{H}, \mathrm{Ar}-\mathrm{H}), 8.8$ (s, $2 \mathrm{H},=\mathrm{NH})$ : El-MS (m/z: RA\% ): $567\left(\mathrm{M}^{+}\right)$. Anal. Calcd for $\mathrm{C}_{25} \mathrm{H}_{17} \mathrm{~N}_{11} \mathrm{O}_{2} \mathrm{~S}_{2} \mathrm{C}: 52.91, \mathrm{H}: 2.99, \mathrm{~N}: 27.16$; found; C:52.77; H:2.82; N:27.03.

\section{$5 d$}

Yellow powder, Yield $62 \%$, M.P. $219^{\circ} \mathrm{C}$. IR $\left(\mathrm{KBr} / \mathrm{cm}^{-1}\right) 3426 \mathrm{~cm}^{-1}(=\mathrm{NH}), 3354 \mathrm{~cm}^{-1}(\mathrm{NH}), 1678$ $\mathrm{cm}^{-1}(-\mathrm{C}=\mathrm{N}-)$ : ${ }^{1} \mathrm{HNMR}\left(400 \mathrm{MHz}, \mathrm{DMSO}-d_{6}\right): \delta=7.0-$ 7.7 (m, 6H, Ar-H), 9.2 (s, 4H, =NH): El-MS (m/z: $R A \%$ ): $665\left(\mathrm{M}^{+}\right)$. Anal. Calcd for $\mathrm{C}_{23} \mathrm{H}_{11} \mathrm{~N}_{11} \mathrm{~S}_{2} \mathrm{Br}_{2}$ $\mathrm{C}: 41.50 ; \mathrm{H}: 1.65$; N:23.15; found; C:.41.34; $\mathrm{H}: 1.51$; $\mathrm{N}: 23.07$
$5 e$

Yellow powder, Yield $72 \%$, M.P. $230^{\circ} \mathrm{C}$. IR $\left(\mathrm{KBr} / \mathrm{cm}^{-1}\right) 3442 \mathrm{~cm}^{-1}(=\mathrm{NH}), 3377 \mathrm{~cm}^{-1}(\mathrm{NH}), 1673$ $\mathrm{cm}^{-1}(-\mathrm{C}=\mathrm{N}-)$ : ${ }^{1} \mathrm{HNMR}\left(400 \mathrm{MHz}, \mathrm{DMSO}-d_{6}\right): \delta=2.3($ $\left.\mathrm{s}, 12 \mathrm{H},-\mathrm{CH}_{3}\right), 6.2-6.6(\mathrm{~m}, 4 \mathrm{H}, \mathrm{Ar}-\mathrm{H}), 8.8(\mathrm{~s}, 4 \mathrm{H},=\mathrm{NH})$ : El-MS (m/z: RA\% ): $563\left(\mathrm{M}^{+}\right)$. Anal. Calcd for $\mathrm{C}_{27} \mathrm{H}_{21} \mathrm{~N}_{11} \mathrm{~S}_{2} \mathrm{C}: 57.54, \mathrm{H}: 3.73, \mathrm{~N}: 27.35$; found; $\mathrm{C}: 57.36, \mathrm{H}: 3.67, \mathrm{~N}: 27.25$.

\section{$5 f$}

Brown powder, Yield $58 \%$, M.P. $236^{\circ} \mathrm{C}$. IR $\left(\mathrm{KBr} / \mathrm{cm}^{-1}\right) 3446 \mathrm{~cm}^{-1}(=\mathrm{NH}), 3350 \mathrm{~cm}^{-1}(\mathrm{NH}), 1680$ $\mathrm{cm}^{-1}(-\mathrm{C}=\mathrm{N}-)$ : ${ }^{1} \mathrm{HNMR}\left(400 \mathrm{MHz}, \mathrm{DMSO}-d_{6}\right): \delta=6.5-$ 7.8 (m, 4H, Ar-H), 9.5 (s, 4H, =NH): El-MS (m/z: RA\% ): $645\left(\mathrm{M}^{+}\right)$. Anal. Calcd for $\mathrm{C}_{23} \mathrm{H}_{9} \mathrm{~N}_{11} \mathrm{~S}_{2} \mathrm{Cl}$ C:42.79, $\mathrm{H}: 1.39, \mathrm{~N}: 23.87$; found: C:42.72, $\mathrm{H}: 1.24, \mathrm{~N}: 23.62$.

$5 g$

Brown powder, Yield $65 \%$, M.P. $210^{\circ} \mathrm{C}$. IR $\left(\mathrm{KBr} / \mathrm{cm}^{-1}\right) 3420 \mathrm{~cm}^{-1}(=\mathrm{NH}), 3340 \mathrm{~cm}^{-1}(\mathrm{NH}), 1675$ $\mathrm{cm}^{-1}(-\mathrm{C}=\mathrm{N}-)$ : ${ }^{1} \mathrm{HNMR}\left(400 \mathrm{MHz}, \mathrm{DMSO}-d_{6}\right): \delta=6.8-$ 7.8 (m, 6H, Ar-H), 9.6 (s, 4H, =NH): El-MS (m/z: RA\% ): $597\left(\mathrm{M}^{+}\right)$. Anal. Calcd for $\mathrm{C}_{23} \mathrm{H}_{11} \mathrm{~N}_{13} \mathrm{O}_{4} \mathrm{~S}_{2} \mathrm{C}: 46.23$, $\mathrm{H}: 1.84, \mathrm{~N}: 30.48$; found; C:46.08, H:1.59, N:30.32.

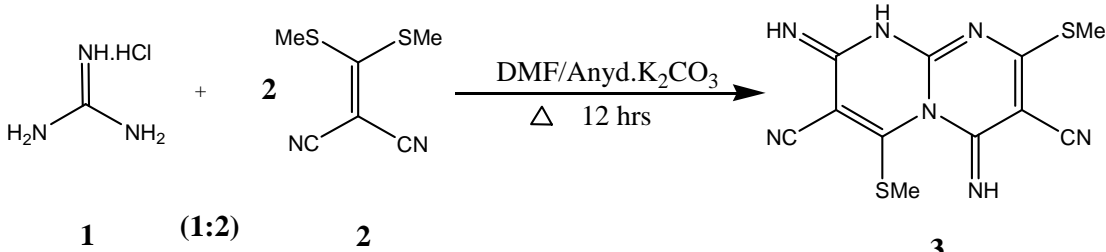

Scheme 1:

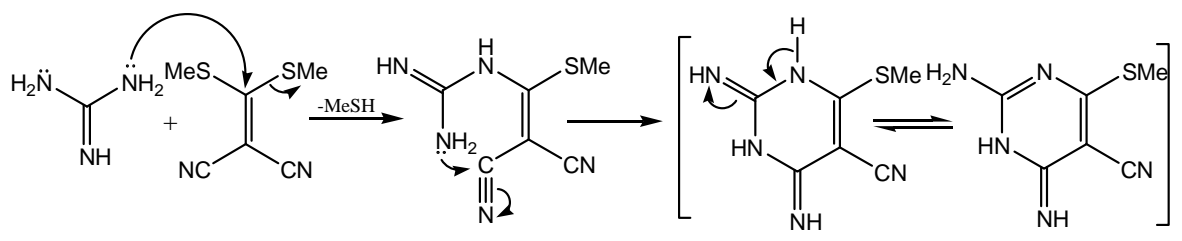

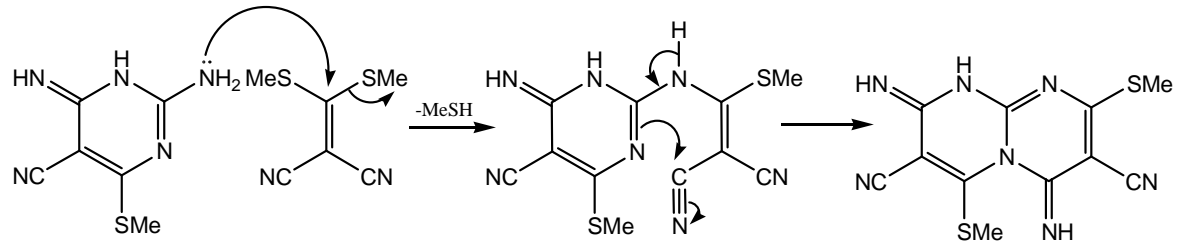

Plausible mechanism of formation of compound (3) 


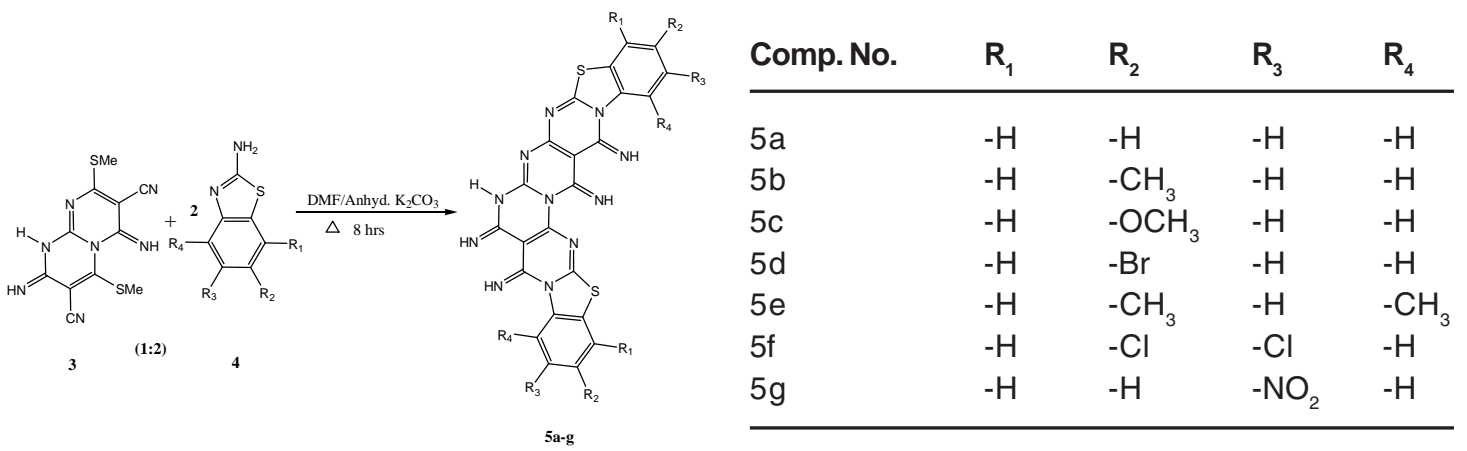

Scheme 2 :

\section{RESULT AND DISCUSSION}

Our approach to synthesize bis (diimino benzothiazolo pyrimido pyrimidines), we have performed efficient reaction to connect guanidine hydrochloride (1) and bis (methylthio) methylene malononitrile (2) were condensed in N,N dimethyl formamide and catalytic amount of anhydrous potassium carbonate to afford (3) Scheme-1

The compound possesses replacable active methyl thio group which is activated by nitrogen atom, electron withdrawing cyano group. When compound (3) (1mole) was condensed independently with 2-amino benzothiazole, 2amino-6-methyl benzothiazole, 2-amino-6-methoxy benzothiazole, 2-amino-6-bromo benzothiazole, 2amino-4,6-dimethyl benzothiazole, 2-amino-5,6dichloro benzothiazole, 2-amino-5-nitro benzothiazole (4) in N,N dimethyl formamide (DMF) and catalytic amount of anhydrous potassium carbonate to obtain diimino bis benzothiazolo pyrimido pyrimidines (5a-g)

The identy of the product was determined by elemental analysis, IR, ${ }^{1} \mathrm{HNMR}$, MASS spectral studies. IR spectram of compound shows absorption band in the region 2250-2180 $\mathrm{cm}^{-1}$ for (CN). ${ }^{1} \mathrm{HNMR}$ spectra of compound exhibit singlet peak in the region of $8.0-9.5 \mathrm{ppm}$ due to $(=\mathrm{NH})$ proton. The MASS spectra shows molecular ion peak which is corresponds to the molecular weight of respective compounds. Spectral studies of all compounds shows that compounds were stable and do not exhibit any tautomerism. The elemental analysis values are in good agreement with theoretical data. All the compounds were screened for their antibacterial and antifungal activities.

Table 1. Antimicrobial activity of compound (5a-g)

\begin{tabular}{|c|c|c|c|c|c|}
\hline \multirow[t]{3}{*}{ Compounds } & \multicolumn{5}{|c|}{ Zone of inhibition in $\mathrm{mm}$} \\
\hline & \multicolumn{4}{|c|}{ Bacteria } & \multirow{2}{*}{$\begin{array}{c}\text { Fungi } \\
\text { Candida Albicus }\end{array}$} \\
\hline & E.Coli & S.typhi & S.aureus & B.subtilis & \\
\hline $5 a$ & - & 08 & 05 & 07 & 09 \\
\hline $5 b$ & 05 & 07 & 09 & 06 & 13 \\
\hline $5 c$ & - & - & 11 & 12 & 12 \\
\hline $5 d$ & 10 & 06 & 15 & 08 & 06 \\
\hline $5 e$ & 08 & 05 & 09 & - & 12 \\
\hline $5 f$ & 16 & 11 & 10 & 13 & 16 \\
\hline $5 g$ & 12 & 13 & 11 & 14 & 12 \\
\hline \multirow[t]{2}{*}{ Positive control } & 18 & 18 & 22 & 20 & 18 \\
\hline & \multicolumn{3}{|c|}{ Streptomycin } & & Amphoterecin B \\
\hline
\end{tabular}




\section{Antimicrobial activity}

The synthesized compounds were evaluated for their antifungal and antibacterial activities against species Candida albicus, E.coli, S.typhy, S.aureus, B.subtilis by paper disk diffusion method. These compounds were dissolved in dimethyl sulphoxide. Incubation period for fungi was 4 days and for bacteria was 24 hours. The newly synthesized compounds shows zone of inhibition $5-18 \mathrm{~mm}$ in diameter where as standard streptomycin exhibit zone of inhibition 18-22 $\mathrm{mm}$ in diameter against E.coli, S.typhi, S.aureus and B.subtilis. Standard amphotericin B shows zone of inhibition $18 \mathrm{~mm}$ in diameter against Candida albicus. Among all the newly synthesized compounds, $5 \mathbf{f}$ (16 1110 $1316 \mathrm{~mm}$ ) and $5 \mathrm{~g}$ (12 $13111412 \mathrm{~mm}$ ) showed higher zone of inhibition against E.coli, S.typhi, S.aureus, B.subtilis and Candida albicus due to presence of di- $\mathrm{Cl}$ and $\mathrm{NO}_{2}$ groups.

\section{CONCLUSION}

In conclusion, we have synthesized diimino pyrimido pyrimidine (3), bis (diimino benzothiazolo pyrimido pyrimidines) (5a-g) have been reported first time and obtained by simple route using mild condition with good yield. These newly synthesized compounds were screened for their antimicrobial activities, compound $5 f$ and $5 \mathrm{~g}$ posseses very good antibacterial and antifungal activity. Hence the importance of such work lies in the possibility that the new compounds might be more effictive drug against bacteria and fungi, which would be helpful in designing more potent antibacterial and antifungal agent for therapeutic use.

\section{ACKNOWLEDGEMENTS}

The authors are grateful to principal, Yeshwant Mahavidyalaya, Nanded, for providing laboratory facilities, and the Director, Indian Institute of Chemical Technology, Hyderabad, for providing spectra.

\section{REFERENCES}

1. Srivastava S. K., Haq W., Chauhan P. M. S., Bioorg. Med. Chem. Lett, 1999, 9, 65.

2. (a) Lunt E, In Barton, D. Ollis, W. Eds , Comprehensive organic chemistry, pergamon press, Oxford, 1974, vol 4, p .493. (b) Brown J D, In Katrizky, A R Rees, C W Eds, Comprehensive Heterocyclic Chemistry, Pergamon Press, Oxferd, 1984, vol 3, p 57.

(c) Clercq E D, Braaerts R J, Biol. Chem, 1987, 262, 14905.

3. Grivski E M, Lee S, Sigel CW, Duch D S and Nichol C A, J. Med. Chem., 1980, 23, 327.

4. Matsumoto $\mathrm{J}$ and Minami S, J. Med. Chem.,1975, 18, 74.

5. Mont N, Teixido J, Borrell J I and Oliver Kappe C, Tetrahedron Lett., 2003, 44, 5385.

6. Oakes $\mathrm{V}$ and Rydon H N, J.Chem. Soc., 1956, 10, 4433.

7. DeGraw J I, Kisliuk R L, Gaumont $Y$ and Baugh C M, J. Med. Chem., 1974, 17, 470.

8. Thompson A M, Rewcastle G W, Bridges A
J, Fry D W, Kraker A J, Denny W A and McMichael A, J. Med. Chem., 1995, 38, 3780 .

9. Coates W J, European patent 351058, Chem. Abstr., 1990, 113, 40711, Ramsey A A. U. S. Patent 3830812 FMC Corp., Chem. Abstr., 1974, 81, 134174.

10. Taylor E C, Knopf R J, Meyer R F, Holmes A, Hoefle M L, J. Am. Chem. Soc.,1960, 82, 5711.

11. Figueroa Villar J D, Carneiro C L, Cruz E R, Heterocycles, 1992, 34, 891.

12. Kitamaru N, Onishi A, European patent 163599, Chem. Abstr., 1984, 104, 186439.

13. Raddatz P, Bergmann R, German patent 360731, Chem. Abstr., 1988, 109, 54786.

14. Rewcastle G W, Bridge A J, Fry D W, Rubin J R, Denny W A, J. Med. Chem., 1997, 40 1820.

15. Sanghvi $Y$ S, Larson S B, Matsumoto S S, Nord L D, Smee D F, Willis R C, Avery T H, Robins R K, Revankar G R, J.Med. Chem., 
1989, 32, 629-637.

16. Tenser R B, Gaydos A K, Hay A, Antimicrob Agents Chemother, 2001, 45, 3657-3659.

17. De la Cruz J P, Carrasco T, Ortega G, Sanchez De la Cuesta F, Lipids, 1992, 27, 192- 194.

18. Sharma P, Rane N, Gurram V K, Bioorg. Med. Chem. Lett., 2004, 14, 4185-4190.

19. Ram V J, Goal A, Sarkhel S, Maulik P R, Bioorg. Med. Chem. Lett.,2002,10, 12751280.

20. Singh A and Bhal A, Ind. J. Chem., 1969, 7, 302.

21. Reimlinger $\mathrm{H}$, Peiren M A, Merenyi R, Chem. Ber., 1975, 108, 3894.

22. Gurinder J S D, Dorcas I O, Scheinmann F, Bates P A, Hursthouse M B, J. Chem. Soc. Perkin Trans I, 1988, 2993.

23. Hataba A A, Fikry R M and Moustafa H Y, J.
Ind. Chem. Soc., 1997, 74, 818.

24. Jimonet $P$, Audiau F, Barreau M, Blanchard J C, Boireau A, BourY, Coleno M A, Doble A, Doerflinger G, Huu C D, Donat $\mathrm{M} \mathrm{H}$, Duchesne J M, Ganil P, Gueremy C, Honore C, Just B, Kerphirique R, Gontier S, Hubert P, Laduron P M, Le Blevec J, Meunier M, Miquet J M, Nemecek C, Pasquet M, Piot O, Pratt J, Ratund J, Reibaud M, Stutzmann J M and Mignani S, J. Med. Chem. 1999, 42, 2828.

25. Sirsat Shivraj B, Vartale Sambhaji P, Organic chem. Curr res, 2012, 1, 5.

26. Sirsat S B, Halikar N K, Kalyankar M B and Vartale S P, journal of pharm. Res, 2012,5, 2700.

27. Sambhaji P Vartale, Shivraj B Sirsat and Nilesh K Halikar, Heterocycl. Commun. 2013, 19 (3), 215-218. 